\title{
Evaluation of patients with polycystic ovarian syndrome at a tertiary care center
}

\author{
Swapna Y.1, V. Srilakshmi ${ }^{2 *}$ \\ ${ }^{1}$ Department of Obstetrics and Gynecology, Deccan College of Medical Sciences, Hyderabad, Telangana, India \\ ${ }^{2}$ Consultant, Area Hospital, Golkonda, Hyderabad, Telangana, India
}

Received: 28 May 2019

Revised: 27 June 2019

Accepted: 02 July 2019

\section{*Correspondence:}

Dr. V. Srilakshmi,

E-mail: giridhar.gollapalli@gmail.com

Copyright: ( $)$ the author(s), publisher and licensee Medip Academy. This is an open-access article distributed under the terms of the Creative Commons Attribution Non-Commercial License, which permits unrestricted non-commercial use, distribution, and reproduction in any medium, provided the original work is properly cited.

\section{ABSTRACT}

Background: It has been estimated that prevalence of PCOS ranges from 5-10\% in reproductive females. Thus, it becomes most common endocrinopathy in this age group. Lack of ovulation and androgen over activity are key features of PCOS. The objective of this study was to patients with polycystic ovarian syndrome at a tertiary care center.

Methods: Present study was following up study with 200 cases of PCOS. Rotterdam criteria were used for the diagnosis of the PCOS. This criterion includes three things. First is woman having in last year $<6$ periods of menstruation. Second is testosterone value $>0.6 \mathrm{ng} / \mathrm{ml}$ and third is each ovary having $>12$ follicles with increased size and volume. Those cases with hypothyroidism, adrenal tumors, congenital adrenal hyperplasia etc were excluded from the present study. Those on steroid therapy were also excluded. Detailed history and clinical examination were carried out for included cases as well as required investigations.

Results: Overall incidence of infertility was found as $27.9 \%$. Out of these 200 cases, 44 (22\%) had PCOS. Majority cases belonged to 20-24 years of age. Hirsutism was most common findings in clinical hyperandrogenism. Risk of insulin resistance syndrome was 4.79 times higher in obesity compared to not obese. Age, BMI, duration of infertility, ovarian volume, and menstrual pattern had effect on ovulation rate following laparoscopic ovarian drilling. Increasing duration of infertility and BMI had lower ovulation rate. Ovulation rate was highest when treated with clomiphene citrate alone than the combination therapy.

Conclusions: Women with obesity have more chances of having PCOS as seen from the present study.

Keywords: Hirsutism, Hyperandrogenism, Insulin resistance, Polycystic ovarian syndrome, Syndrome

\section{INTRODUCTION}

It has been estimated that prevalence of PCOS ranges from $5-10 \%$ in reproductive females. Thus, it becomes most common endocrinopathy in this age group. Lack of ovulation and androgen over activity are key features of PCOS.

Whenever anovulation is there, there are disturbances of the menstruation, in the form of complete absence of menses or reduced degree of menses. In cases of hyperandrogenism, there is growth of facial hairs; increased incidence of acne, loss of hairs in androgenic fashion, there is increased levels of the testosterone. ${ }^{1}$

Women with PCOS tend to be obese. They can also have insulin resistance. Insulin resistance can be seen in underweight women with PCOS. Not only testosterone is increased but also the luteinizing hormone levels are also increased. At the same time, there is decrease in the production of sex hormone-binding globulin (SHBG). ${ }^{2}$ 
One of the most consistent features of PCOS is polycystic nature of ovaries which can be detected by ultrasonography. But it has been said that even in women with apparent no PCOS, one fifth of them may have polycystic ovaries. It is not easy to detect PCOS. Hence Rotterdam gave the criteria that out of three, if any two are present, then the women can be labeled as having PCOS. First is woman having in last year $<6$ periods of menstruation. Second is testosterone value $>0.6 \mathrm{ng} / \mathrm{ml}$ and third is each ovary having $>12$ follicles with increased size and volume. ${ }^{3}$

In women with PCOS, there is presence of the insulin resistance. This leads to the increased levels of insulin in the blood. This leads to "ovarian hyperandrogenism". This leads to various manifestations of PCOS as described before. ${ }^{4}$

Apple shape obesity is common feature of android obesity. Pear shape obesity is common feature of gynoid obesity. In women with PCOS, android obesity is commonly seen. Obesity leads to increased intensity of the resistance of the insulin. It is commonly found to be associated with infertility and other features. ${ }^{5}$ In women with PCOS, there is resistance to the action of insulin. This coupled with hyperinsulinemia causes increase in the triglycerides, LDL, VLDL and a decrease in the HDL, increase in the blood pressure levels. Women with PCOS are at an increased risk of developing diabetes and other non-communicable diseases. ${ }^{6,7}$ Present study was carried out to study the incidence of PCOS among the various causes of infertility and to establish the fact that the operative Triple Puncture Video Laparoscopy has a very important role to play in the management of PCOD and it has the additional advantage of offering therapeutic measures at the same sitting.

\section{METHODS}

The Study design is Present study was following study with Sample size: 200 cases of PCOS. The Criteria used Rotterdam criteria were used for the diagnosis of the PCOS. This criterion includes three things. First is woman having in last year $<6$ periods of menstruation. Second is testosterone value $>0.6 \mathrm{ng} / \mathrm{ml}$ and third is each ovary having $>12$ follicles with increased size and volume. The Study period was September 2011 to October 2012. The Settings was Department of Obstetrics and Gynecology, Deccan College of Medical Sciences, Hyderabad, Telangana, India.

\section{Inclusion criteria}

- Patients fitting in Rotterdam criteria,

- Willing to participate.

\section{Exclusion criteria}

- Patients with co-morbidities like adrenal tumors, hypothyroidism, OC pill use, use of glucocorticoids, use of anti-androgen drugs, use of drugs for diabetes,

- $\quad$ Not willing to participate.

Data was recorded regarding history, examination; investigations in a pre-designed, pretested, semi structured study questionnaire designed for the present study after the cases gave their informed consent.

Height and weight were measured as per the standard guidelines and protocol. Body mass index was calculated based on height and weight. Waist and hip circumference were measured as per the standard guidelines and protocol. Waist hip ratio was calculated based on the waist and hip circumference.

Presence of symptoms pertaining to PCOS were asked and noted. Patients were asked to give the fasting blood samples in the morning on any day from second to fifth day of the menstrual cycle. Double anti body RIA method was used for the determination of the fasting insulin levels. Glucose oxidase method was used for the determination of the fasting glucose levels. Other investigation like levels of $\mathrm{LH}, \mathrm{FSH}$ and TSH were carried out. Curvilinear transducer with frequency 3.5 megahertz was used in trans-abdominal ultrasonography.

\section{Statistical analysis}

The data was entered in the master chart of the excel sheet and analyzed using proportions and appropriate statistical test was applied as and when required.

\section{RESULTS}

Table 1 shows incidence of infertility and the variability in clinical phenotypes characterizing PCOS cases. The overall incidence of infertility was found out to be $27.9 \%$ in the present study. Out of these 3170 cases with infertility, 200 cases were included in the present study. Out of these 200 cases, 44 had PCOS i.e. 22\%. 80 cases had ologo ovulation i.e. 40\%. 18 cases had hyper and rogenism i.e. 9\%. 18 cases had PCOS plus chronic anovulation i.e. $9 \%$. 14 cases had hyperandrogenism plus PCOS i.e. 7\%. 10 cases had hyperandrogenism plus chronic anovulation i.e. 5\%. 16 cases had hyperandrogenism plus chronic anovulation+PCOS i.e. $8 \%$.

Table 2 shows demographic details of cases studied. Majority of the cases belonged to the age group of 20-24 years of age. This was followed by $25 \%$ of the cases who belonged to the age group of 25-29 years of age. This was followed by $21.5 \%$ of the cases who belonged to the age group of less than 19 years of age. Only $12.5 \%$ of the cases belonged to the age group of more than 30 years of age. $55 \%$ of the cases were found out to be overweight. $38 \%$ of the cases were found to have grade 1 obesity. Only $7 \%$ of the cases were found out to be having obesity grade 2 . 
Table 3 shows clinical Hyperandrogenism among the study subjects. 48 cases i.e. $24 \%$ of the cases were found to have acne. 130 cases i.e. $65 \%$ of the cases were found to be suffering from hirsutism. 12 cases i.e. $6 \%$ of the cases were found to be suffering from androgenic alopecia. 10 cases i.e. $5 \%$ of the cases were found to have acanthosis nigricans. Thus, hirsutism was the most common findings in clinical hyperandrogenism among the study subjects in the present study.

Table 1: Incidence of infertility and the variability in clinical phenotypes characterizing PCOS cases.

\begin{tabular}{|lll|}
\hline Total gynec patients & Total patients with infertility & Incidence of infertility \\
\hline 11350 & 3170 & 27.9 \\
\hline Criteria & No. of patients $(\mathrm{n}=200)$ & $\%$ \\
\hline PCOS & 44 & 22 \\
\hline Oligo ovulation & 80 & 40 \\
\hline Hyper androgenism & 18 & 9 \\
\hline PCOS+Chronic anovulation & 18 & 9 \\
\hline Hyperandrogenism+PCOS & 14 & 7 \\
\hline Hyperandrogenism+Chronic anovulation & 10 & 5 \\
\hline Hyperandrogenism+Chronic anovulation+PCOS & 16 & 8 \\
\hline
\end{tabular}

Table 2: Demographic details of cases studied $(n=200)$.

\begin{tabular}{|llll|}
\hline Demographic characteristics & Number & $\%$ \\
\hline \multirow{3}{*}{ Age (years) } & $<19$ & 43 & 21.5 \\
\cline { 2 - 4 } & $20-24$ & 83 & 41 \\
\cline { 2 - 4 } & $25-29$ & 50 & 25 \\
\cline { 2 - 4 } & $>30$ & 25 & 12.5 \\
\hline \multirow{2}{*}{$\begin{array}{l}\text { Body mass } \\
\text { index }\left(\mathrm{kg} / \mathrm{m}^{2}\right)\end{array}$} & $<29$ & 110 & 55 \\
\hline \multirow{2}{*}{$\begin{array}{l}\text { Menstrual } \\
\text { cycles }\end{array}$} & $30-34$ & 76 & 38 \\
\cline { 2 - 4 } & $>34$ & 84 & 7 \\
\hline
\end{tabular}

Table 3: Clinical Hyperandrogenism among the study subjects.

\begin{tabular}{|lll|}
\hline Characteristics & No. of Patients $(\mathbf{n}=200)$ & $\%$ \\
\hline Acne & 48 & $24 \%$ \\
\hline Hirsutism & 130 & $65 \%$ \\
\hline Androgenic Alopecia & 12 & $6 \%$ \\
\hline Acanthosis Nigricans & 10 & $5 \%$ \\
\hline
\end{tabular}

Table 4 shows insulin resistance in non-obese \& obese PCOS. It was found that the prevalence of insulin resistance syndrome was $70 \%$ among those who were obese compared to $32.5 \%$ of insulin resistance syndrome among those who were not obese. This difference in the insulin resistance syndrome among the obese cases and the non-obese cases was found out to be statistically significant. On calculation of the odds ratio, it was found that the risk of insulin resistance syndrome was 4.79 times higher in those with obesity compared to those who were not obese with $95 \%$ confidence interval of 2.62 8.75 .

Table 5 shows ovulation rates in PCOS patients who underwent laparoscopic ovarian drilling (LOD). These 200 patients who were diagnosed by Rotterdam's criteria as PCOS were further evaluated by diagnostic laparoscopy and underwent ovarian drilling at the same sitting. They were followed up in the outpatient clinic for ovulation by TVS. It was found that age, BMI, duration of infertility, ovarian volume, and menstrual pattern had effect on ovulation rate following laparoscopic ovarian drilling. It was also found that increasing duration of infertility and BMI had lower ovulation rate.

Table 4: Insulin resistance in non-obese and obese PCOS.

\begin{tabular}{|c|c|c|c|c|c|c|c|c|}
\hline \multirow[t]{2}{*}{ Obesity } & \multicolumn{2}{|c|}{$\begin{array}{l}\text { Insulin resistance } \\
\text { present }\end{array}$} & \multicolumn{2}{|c|}{ Insulin resistance absent } & \multirow{2}{*}{$\begin{array}{l}\text { Yate's } \\
\text { corrected chi } \\
\text { square }\end{array}$} & \multirow{2}{*}{ p value } & \multirow{2}{*}{$\begin{array}{l}\text { Odds } \\
\text { Ratio }\end{array}$} & \multirow{2}{*}{$\begin{array}{l}95 \% \\
\text { confidence } \\
\text { interval }\end{array}$} \\
\hline & Number & $\%$ & Number & $\%$ & & & & \\
\hline Yes & 63 & 70 & 27 & 30 & \multirow{2}{*}{26.04} & \multirow{2}{*}{$<0.0001$} & \multirow{2}{*}{4.79} & \multirow{2}{*}{$2.62-8.75$} \\
\hline No & 36 & 32.5 & 74 & 67.5 & & & & \\
\hline
\end{tabular}


Table 6 shows effect of clomiphene citrate in combination with different drugs in medical therapy. The rate of ovulation was $64 \%$ among those who were treated with Clomiphene citrate.

The rate of ovulation was 59\% among those who were treated with Clomiphene citrate+metformin. The rate of ovulation was $45 \%$ among those who were treated with Clomiphene citrate+pioglitazone.

Thus, the ovulation rate was highest when treated with clomiphene citrate alone than the combination therapy. The rate of pregnancy was $9 \%$ among those who were treated with Clomiphene citrate. The rate of pregnancy was $13.2 \%$ among those who were treated with Clomiphene citrate+metformin. The rate of pregnancy was $12 \%$ among those who were treated with Clomiphene citrate+pioglitazone.

Table 5: Ovulation rates in PCOS patients who underwent laparoscopic ovarian drilling (LOD).

\begin{tabular}{|llll|}
\hline Characteristics & Category & Sive $\mathbf{n = 2 0 0}$ & $\begin{array}{l}\text { Ovulation } \\
\text { rate }\end{array}$ \\
\hline Age in years & $<30$ years & 175 & $78 \%$ \\
& $>30$ years & 25 & $70 \%$ \\
\hline Body mass & $<29$ & 110 & $81 \%$ \\
index $\left(\mathrm{kg} / \mathrm{m}^{2}\right)$ & $30-34$ & 76 & $84 \%$ \\
& $>34$ & 14 & $44 \%$ \\
\hline Duration of & $<3$ years & 154 & $90 \%$ \\
Infertility & $3-6$ years & 30 & $83 \%$ \\
Ovarian & $>6$ years & 16 & $52 \%$ \\
Volume & $<14$ & 166 & $80 \%$ \\
\hline
\end{tabular}

Table 6: Effect of clomiphene citrate in combination with different drugs in medical therapy.

\begin{tabular}{|lll|}
\hline Drugs used & $\begin{array}{l}\text { Ovulation } \\
\text { in \% }\end{array}$ & $\begin{array}{l}\text { Pregnancy } \\
\text { in \% }\end{array}$ \\
\hline Clomiphene citrate & 64 & 9 \\
\hline $\begin{array}{l}\text { Clomiphene citrate }+ \\
\text { metformin }\end{array}$ & 59 & 13.2 \\
\hline $\begin{array}{l}\text { Clomiphene citrate }+ \\
\text { pioglitazone }\end{array}$ & 45 & 12 \\
\hline
\end{tabular}

\section{DISCUSSION}

In the present study the incidence of infertility was $12.4 \%$. The incidence of PCOS among these infertile patients was $10.5 \%$. Dasgupta $S$ et al, reported that 7 $10 \%$ of infertile women patients as suffering from PCOS. ${ }^{8}$

In the present study, 127 had primary infertility and 73 had secondary infertility with PCOS. It was found that majority of the primary infertility with PCOS were from the age group of 20-25 years and secondary PCOS cases were in the age group of 25-30 years. It was found that
110 patients were overweight, 76 patients were grade one obese and 14 patients were grade two obese. PCOS women with BMI $>34 \mathrm{~kg} / \mathrm{m} 2$ achieved significantly lower rates of ovulation after LOD compared with moderately overweight and normal overweight women.

The findings in the present study are in agreement with the previous reports by Gjonnaess $\mathrm{H}$ et al, who reviewed 252 patients who underwent LOD and found that women with marked obesity achieved significantly lower ovulation rates compared with women with normal and moderately elevated BMI. ${ }^{9}$

Hence weight reduction by decrease of diet and regular exercise was advised as the first line of treatment in obese patients with PCOS. This showed an increase in ovulation and pregnancy rates. This significant outcome with weight reduction can be compared to studies carried out by Moran LJ et al, where $44 \%$ increase in ovulation rates was seen with a daily diet of $600 \mathrm{JK} /$ day and weight loss of $7.7 \mathrm{~kg}$. $^{10}$

In the present study, the menstrual disorder of the sample, 76 women had regular cycles, 106 had oliogomenorrhea and amenorrhea was seen in 18 patients. Menstrual irregularities like oligomenorrhea and amenorrhea was seen in 18 patients. Menstrual irregularities like oligomenorrhea and amenorrhea were seen in association with chronic anovulation with PCOS by Frank S et al, and Goldzeiher JW et al. ${ }^{11,12}$

The incidence of oligomenorrhea in the present study was $53 \%$ which is comparable to studies done by Frank S et al, and Goldzeiher JW et al. ${ }^{11,12}$

Hyper androgenic features like acne was seen in $24 \%$ of the cases in the present study which is comparable to study by Frank S et al, Hirsutism was seen in $65 \%$ of the cases which is comparable to study report by Frank $\mathrm{S}$ et al, and Goldzeiher JW et al. ${ }^{11,12}$ Insulin resistance was seen in $32.7 \%$ in non-obese and $70 \%$ of obese PCOS in the present study, which is in accordance with Glueck CJ et al, who reported that Insulin resistance was seen in $28 \%$ in non-obese and $75 \%$ of obese PCOS. ${ }^{13}$

These patients have shown marked improvement when advised for weight reduction and exercise. The percentage of ovulation and pregnancy was found to be $64 \%$ and $9 \%$ respectively in patients with PCOS after clomiphene citrate administration which can be compared to the studies conducted by Hammond $\mathrm{G}$ et al. ${ }^{14}$

Insulin sensitizers like metformin and pioglitazone when administered along with clomiphene citrate have shown to increase the ovulation rates by $59 \%$ and $45 \%$ respectively and pregnancy rates by $13.2 \%$ and $12 \%$ respectively which is comparable to the studies conducted by Zain MM et al, who reported that the ovulation rates with clomiphene citrate and clomiphene citrate plus metformin were $59 \%$ and $68.4 \%$ and the 
pregnancy rates were $15.4 \%$ and $18.4 \%$ respectively. ${ }^{15}$ PCOS was the most common cause of anovulatory infertility being found in $75 \%$ of the cases as per Hull MGR et al. ${ }^{16}$ Laparoscopic ovarian drilling (LOD) has been widely used to induce ovulation in PCOS women with failure of treatment with clomiphene citrate.

Many authors have reported high ovulation rates of up to $80 \%$ following LOD like the one Gjonnaess $\mathrm{H}$ et al. ${ }^{9}$ It was found that age less than 30 years responded well to LOD and had spontaneous ovulation rate of $78 \%$ and age of more than 30 years also ovulated spontaneously in the present study. This showed that increasing age has not much effect onovulaiton induction by LOD.

\section{CONCLUSION}

All patients presenting with complaint of infertility should be evaluated for menstrual irregularities like oligomenorrhea and amenorrhea, symptoms of hyperandrogenism like acne, androgenic alopecia, hirsutism and acanthosis nigricans, insulin resistance to be diagnosed as PCOS. Incidence of menstrual irregularities is high in women with PCOS. All women diagnosed to have PCOS should be evaluated for insulin resistance specifically if they are obese.

Funding: No funding sources

Conflict of interest: None declared

Ethical approval: The study was approved by the Institutional Ethics Committee

\section{REFERENCES}

1. Adams J, Polson DW, Abdulwahid N, Morris DV, Franks S, Mason HD, et al. Multifollicular ovaries clinical and endocrine response. Lancet. 1985;2(8469-70):1375-8.

2. Adams J, Polson DW, Franks S. Prevalence of polycystic ovarian syndrome in women with anovulation and idiopathic hirsutism. $\mathrm{Br}$ Med J. 1986;293(6543):355-9.

3. Bailey CJ, Turner RC. Metformin. N Engl J Med. 1996;334(9):574-9.

4. Balen AH, Conway GS, Kaltsas G, Techatrasak K, Manning PJ, West $\mathrm{C}$ et al. Polycystic ovary syndrome. The spectrum of the disorder in 1741 patients. Hum Reprod. 1995;10(8):2107-11.

5. Dunaif A1, Segal KR, Shelley DR, Green G, Dobrjansky A, Licholai T. Evidence for distinctive and intrinsic defects in insulin action in polycystic ovarian syndrome. Diabetes. 1992;41(10):1257.

6. Dunaif A. Insulin resistance and the polycystic ovarian syndrome; mechanism and implication for pathogenesis. Endocr Rev. 1997;18(6):774-800.

7. Franks S. Diagnosis of polycystic ovary syndrome. In defense of the Rotterdam criteria. J Clin Endocrinol Metab. 2006;91(3):786-9.

8. Dasgupta S, Mohan Reddy B. Present status of understanding on the genetic etiology on polycystic ovarian syndrome. J Postgrad Med. 2008;54(2):11525 .

9. Gjonnaess H. Polycystic ovarian syndrome treated by ovarian electrocautery through the laparoscope. Fertile Steril. 1984;41(1):20-5.

10. Moran LJ, Noakes M, Clifton PM et al. Dietary composition in restoring reproductive and metabolic physiology in overweight women with polycystic ovarian syndrome. J Clin Endocrinol Metab. 2003;88(2):812-9.

11. Franks S. Polycystic ovary syndrome. N Engl J Med. 1995;333(13):853-61.

12. Goldzeiher JW. Polycystic ovarian disease. Fertile Steril. 1981;35(4):371-94.

13. Glueck CJ1, Dharashivkar S, Wang P, Zhu B, Gartside PS, Tracy Tet al. The obesity and extreme obesity, manifest by ages 20-24 years, continuing through 32-41 years in women, should alert physicians to the diagnostic likelihood of polycystic ovary syndrome as a reversible underlying endocrinopathy. Eur J Obstet Gynecol Reprod Biol. 2005;122(2):206-12.

14. Hammond M, Halme J, Talbert L. Factors affecting the pregnancy rate in clomiphene citrate induction of ovulation. Obstet Gynecol. 1983;62(2):196-202.

15. Zain MM, Jamaluddin R, Ibrahim A, Norman RJ. Comparison of clomiphene citrate, metformin or the combination of both for first line ovulation induction, achievement of pregnancy and live birth in Asian women with polycystic ovary syndrome. A randomized controlled trial. Fertile Steril. 2009;91(2):514-21.

16. Hull MGR. Polycystic ovarian disease. Clinical aspect and prevalence. Res Clin Forums. 1989;11:21.

Cite this article as: Swapna Y, Srilakshmi V. Evaluation of patients with polycystic ovarian syndrome at a tertiary care center. Int J Reprod Contracept Obstet Gynecol 2019;8:3327-31. 